\title{
Sangrado mayor en paciente adulto, Testigo de Jehová
}

\section{Massive blood loss in adult jehovah's witness patient}

\author{
Daniel Rivera Tocancipá1, Carlos Andrés Rivera Ortiz ${ }^{2}$
}

Palabras clave: Testigos de Jehová, sangrado mayor, pérdida masiva de sangre, choque hipovolémico, transfusiones, hemoderivados.

Key words: Jehovah's Witnesses, major bleeding, massive blood loss, hypovolemic shock transfusions, blood products.

\section{Resumen}

El sangrado mayor (SM) se define como la pérdida de la volemia en un período de tiempo inferior a 24 horas o la pérdida del $50 \%$ de la volemia en un lapso de tiempo inferior a 3 horas en un individuo previamente sano. Teniendo en cuenta la firme posición a rechazar cualquier transfusión de sangre o sus componentes por parte de los Testigos de Jehová, el manejo del sangrado mayor en ellos es un reto ya sea en los servicios de urgencias o de cirugía, con serias repercusiones éticas y legales. El anticiparse al sangrado mayor en Testigos de Jehová permite instaurar acciones preventivas al choque hipovolémico. Para el tratamiento de pacientes Testigos de Jehová con sangrado mayor, se proponen metas terapéuticas que incluyen la realización de intervenciones hemostáticas o farmacológicas durante el tratamiento quirúrgico y en el posoperatorio, plantear metas hemodinámicas y de ventilación mecánica apropiadas. Se hace una revisión narrativa de la literatura de los últimos 10 años y se exponen las acciones preventivas, las metas terapéuticas y las diferentes intervenciones de las que se dispone actualmente para enfrentar el SM.

\begin{abstract}
Major bleeding (MB) is defined as the loss of volume over a less-than-24hour period of time or the loss of $50 \%$ of volume in a less-tha-3-hour time in a previously healthy individual. Considering the firm determination to reject any transfusion of blood or blood components on the part of Jehovah's Witnesses, the management of major bleeding in them is a challenge whether in emergency room or surgery services. This fact has serious ethical and legal implications. Preventing major bleeding in Jehovah's Witnesses allows the introduction of preventive measures to hypovolemic shock. For the treatment of Jehovah's Witness patients having major bleeding, therapeutic targets are proposed including the implementation of hemostatic or pharmacological interventions during surgical treatment; likewise in the postoperative period it is proposed appropriate hemodynamic and mechanic ventilation goals. It is a narrative review of the last 10 -year literature and preventive actions, therapeutic goals and different interventions currently at disposal to deal with $\mathrm{MB}$ are presented.
\end{abstract}

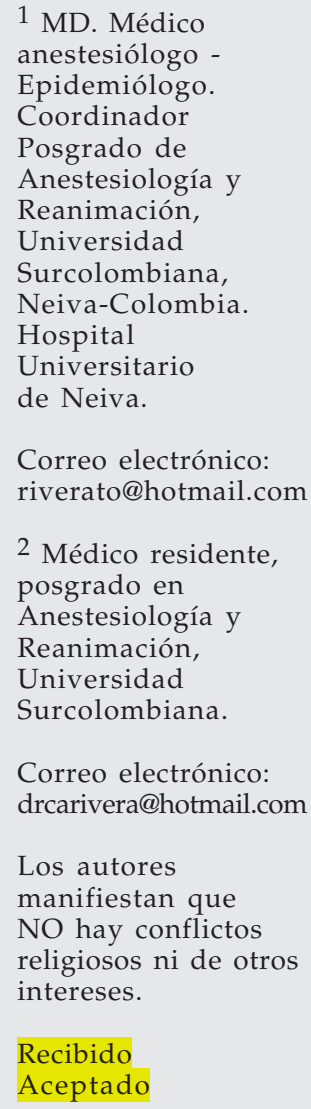

\section{Introducción}

Las raíces de los Testigos de Jehová se remontan a 1870, en Pittsburgh, Pensilvania, Estados Unidos, por Charles Taze Russell.
Se estima que en el mundo existen alrededor de seis millones de personas practicantes de esta fe y en Colombia aproximadamente $120.000^{(1-3)}$. Su doctrina se basa en interpretaciones bíblicas. Uno de sus principios es 
la no aceptación a recibir trasfusiones de sangre aunque de esto dependa la vida propia o de sus familiares, fundamentados en la interpretación bíblica que expresa: No comeréis la sangre de ninguna carne, porque la vida de toda carne es su sangre, cualquiera que la comiere será exterminado Génesis 9 v4. La sangre es la vida; así que no deben comer la vida junto con la carne. Levítico 7 v25. Ninguno de ustedes podrá comer sangre, tampoco aquellos que vivan lejos de ti podrán comerla Levítico $17 v 12^{(4)}$.

Con esta premisa el abordaje médico-quirúrgico de los pacientes Testigos de Jehová no es fácil y conjuga, además, aspectos éticos y legales que dificultan el ejercicio del clínico al momento de tomar decisiones que impliquen la utilización de componentes sanguíneos.

La relación médico-paciente, se basa bajo tres principios éticos:

\section{- Principio de autonomía}

Establece que el paciente tiene el derecho a participar en las decisiones que se tomen sobre su enfermedad y la aceptación o no de los diferentes tratamientos propuestos. Por ello se ha establecido la firma del consentimiento informado como garante de este principio, respetándole al enfermo el uso de sus libertades, deseos y creencias, que en ocasiones no se correlacionan con las metas clínicas del médico tratante ${ }^{(5)}$.

- Principio de beneficencia

Es la intención del médico de querer hacer el bien y prevenir cualquier daño innecesario derivado de una intervención médica. Debe respetar el principio de autonomía ${ }^{(5)}$

\section{- Principio de no maleficencia}

Hace referencia explícita a la intención de no hacer daño (tanto físico como psicológico) por parte del médico. Se podría considerar que transfundir componentes sanguíneos a Testigos de Jehová que previamente hayan manifestado sus deseos de no aceptar terapia con hemoderivados, configure un daño moral al enfermo ${ }^{(5)}$.

El paciente Testigos de Jehová enfoca su situación desde la concepción religiosa, su visión de la vida eterna y el vínculo con
Dios para la toma de decisiones sobre el tratamiento de su enfermedad. El médico antepone razones fisiopatológicas, epidemiológicas y farmacológicas, para sustentar las intervenciones terapéuticas. Los dos enfoques frecuentemente se anteponen, al menos en lo referente a la transfusión sanguínea y es ahí donde respetando el principio de autonomía el médico debe plantear alternativas de tratamiento. La controversia en estos casos, con implicaciones éticas profundas como en menores de edad, ha llegado incluso al ámbito legal y al respecto la corte constitucional en sentencia de Agosto de 2002 establece:

1. En los casos que trate de menores de edad con padres Testigos de Jehová, que rechacen la terapia con hemoderivados hacia su hijo, se establece que el derecho a la vida en el indefenso menor de edad prima sobre las creencias religiosas de los padres y se autoriza al médico a realizar transfusiones sanguíneas ${ }^{(6)}$.

2. En los casos de los menores de edad adultos, llamados "menor adulto", se establece que estos pacientes Testigos de Jehová pueden participar en la toma de decisiones clínicas que lo afecten, pero se establece que por el hecho de ser menores de edad no son plenamente capaces por disposición de la ley para asumir este tipo de decisiones, por lo que prevalece la decisión de los padres en el caso de que la intención de estos sea la autorización para que sea practicada la terapia con transfusiones de sangre, teniendo en cuenta que la voluntad está dirigida a proteger la vida de su hijo ${ }^{(6)}$.

3. En los casos de los pacientes Testigos de Jehová que sean mayores de edad, plenamente capaces de asumir decisiones, prima el principio de autonomía y debe respetarse su decisión siempre que con ella no cause daño a terceros o a la colectividad. Habilitar al médico para imponerle su criterio al paciente, sería tanto como despojar al individuo de su autonomía, trasladándola a otro en razón de su calificación profesional, lo que es inadmisible en la concepción de hombre que subyace en este tipo de organización política"(6).

Es claro con razones éticas y legales que el médico debe aceptar y respetar las deci- 
siones tomadas por el paciente así estas vayan en contra de los ideales del galeno, con excepción de la población menor de edad.

En aquellos casos en los que el escenario de una situación clínica no amenace de forma inminente la vida de un paciente Testigo de Jehová, el médico tiene el derecho de poder abstenerse de brindar atención médica sin ninguna implicación médico legal y en estos casos la mejor conducta es ayudar al paciente a encontrar un profesional que acepte las condiciones del enfermo y tenga experiencia en los programas de medicina sin sangre.

\section{Sangrado mayor y metas terapéuticas}

1.1 Sangrado mayor: es definido como la pérdida de la volemia en un periodo inferior a 24 horas o la pérdida del $50 \%$ de la volemia en un lapso de tiempo inferior a 3 horas en un individuo previamente sano. Se estima la volemia del adulto en el $7 \%$ de su peso corporal ideal y en la población pediátrica del 8 al 9\% del peso ideal. Se han descrito otras definiciones como una tasa de perdida de sangre de $150 \mathrm{ml} /$ minuto $^{(7)}$. Una aproximación del grado de sangrado con que se presenta el paciente nos la presenta la American College of Surgeons Advanced Trauma Life Support classification of haemorrhage severity descrita en la Tabla $1^{(9)}$. El escenario típico del paciente con sangrado mayor es en trauma mayor (usualmente requiere cirugía) y la cirugía electiva mayor. Por tal razón estas medidas deben ser instauradas y mantenidas por el anestesiólogo.

1.2 Metas terapéuticas: reconociendo rápidamente el paciente en riesgo de sangrado mayor se podrán instaurar acciones tempranas que permitan prevenir el choque hipovolémico o manejar mejor sus consecuencias si se presenta. En tal sentido se propone $^{(7,8)}$ :

a. Adecuar el uso de terapias farmacológicas y productos sanguíneos alternos en caso de ser aceptados por el paciente Testigo de Jehová.

b. Implementar medidas de ahorro de sangre.

c. Detener la hemorragia de tratamiento quirúrgico.

d. Mantener perfusión y oxigenación tisular.

e. Tratar la causa del sangrado de etiología médica.

Es importante que los médicos tratantes se encuentren dispuestos a respetar el ejercicio de esta creencia religiosa y proporcionen las mejores alternativas médicas a su alcance. Los Testigos de Jehová permiten la utilización de medidas farmacológicas y en algunas instancias, incluso podrían aceptar la utilización de algunos componentes sanguíneos bajo condiciones específicas; es aquí

Tabla 1. American College of Surgeons Advanced Trauma Life Support Classification of Haemorrhage Severity

\begin{tabular}{lcccc}
\hline $\begin{array}{l}\text { Severidad de la Hemorragia } \\
\text { de acuerdo a la clasificación }\end{array}$ & Clase I & Clase II & Clase III & Clase IV \\
\hline ACS/ATLS & $<750$ & $750-1.500$ & $1.500-2.000$ & $>2.000$ \\
Pérdida de sangre (ml) & $<100$ & $>100$ & $>120$ & $>140$ \\
Frecuencia cardiaca & Normal & Normal & Disminuido & Disminuido \\
Frecuencia de pulso (por minuto) & Normal & Disminuido & Disminuido & Disminuido \\
Presión sanguínea (mm Hg) & $14-20$ & $20-30$ & $30-40$ & $>40$ \\
Frecuencia respiratoria (por minuto) & $>30$ & $20-30$ & $5-15$ & $<5$ \\
$\begin{array}{l}\text { Gasto urinario (ml/hora) } \\
\begin{array}{l}\text { Sistema Nervioso Central } \\
\text { (estado mental) }\end{array}\end{array}$ & $\begin{array}{c}\text { Gran } \\
\text { ansiedad }\end{array}$ & $\begin{array}{c}\text { Discretamente } \\
\text { ansioso }\end{array}$ & $\begin{array}{c}\text { Ansioso, } \\
\text { confuso }\end{array}$ & Letárgico \\
\hline
\end{tabular}

Valores estimados para un adulto de $70 \mathrm{Kg}$. 
en donde el consentimiento informado juega un papel fundamental, como una constancia clínica, que evidencia y da el soporte legal hacia el rechazo o la aceptación de la utilización de cada terapia.

Podemos enfrentarnos a pacientes Testigos de Jehová con posiciones ambiguas y con una gran variedad de posturas frente a la utilización o no de componentes sanguíneos. Algunos, por ejemplo, rechazan incluso la utilización de cristaloides o por el contrario, otros aceptan medidas terapéuticas que incluyan la utilización de componentes sanguíneos bajo escenarios clínicos determinados como la hemodilución normovolémica aguda sin que la sangre se desconecte nunca del circuito que va al paciente permitiendo así la reutilización de su sangre. Por esta razón existen tratamientos clasificados en tres categorías:

a. Tratamiento aceptable.

b. Decisión personal (aceptable para algunos, inaceptable para otros).

c. Tratamiento inaceptable (Tabla 2) ${ }^{(10)}$.

De tal manera que la aceptación o no de diferentes intervenciones clínicas son finalmente individuales y así debe ser nuestro abordaje: individualizado.

Tabla 2. Posición religiosa frente a las terapias médicas

Tratamiento aceptable (aceptable vs. inaceptable)

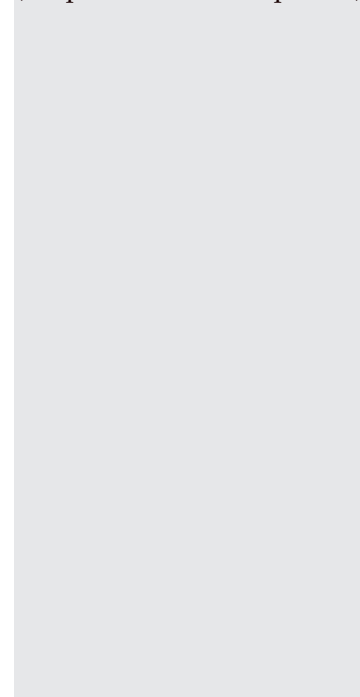

Tratamiento inaceptable
- La mayoría de técnicas quirúrgicas y anestésicas para la conservación de sangre. (Instrumentos hemostáticos quirúrgicos, hipotensión controlada, anestesia regional, cirugía mínimamente invasiva.

- Procedimientos diagnósticos y terapéuticos. (Flebotomía para test de laboratorio, embolización angiográfica).

- Agentes farmacológicos que no contengan componentes sanguíneos o derivados. - Medicamentos que favorezcan la coagulación: ácido tranexámico, ácido epsilon aminocaproico, aprotinina, desmopresina, factor viia recombinante, estrógenos conjugados.

- Factores de crecimiento hematopoyético y hematínicos. (Albúmina, Eritropoyetina, Hierro).

- Productos recombinantes. (Factores de la Coagulación).

- Transportadores sintéticos de oxígeno. (Hemoglobin-base oxygen carrier (HBOC) o Perfluorocarbonos).

- Expansores de volumen no sanguíneos. (SS 0,9\%, Lactato Ringer, Hydroxyethyl Starches).

- Técnicas Salvadoras de células sanguíneas. (Autotransfusión perioperatoria o posoperatoria.

- Hemodilución Normovolémica Aguda Intraoperatoria.

- Secuestro de Componentes Sanguíneos Autólogos Perioperatorios. (Plaquetoferesis, Preparación de gel de Fibrina, Gel de Plaquetas, Plasma rico en Plaquetas.

- Bypass cardiopulmonar.

- Aféresis.

- Hemodiálisis.

- Fracciones derivados del plasma. (Inmunoglobulinas, vacunas, albúmina, crioprecipitados).

- Fracciones de Sangre que contengan Productos hemostáticos. (Factores Concentrados de la Coagulación, Complejo Concentrado de Protrombina, Sellantes de Fibrina, Bandas Hemostáticas que contengan Fracciones de Plasma, Sellantes de Trombina.

- Productos que contengan Fracciones de Sangre derivados del Plasma. (Albúmina Sérica Humana, Eritropoyetina, Estreptoquinasa, G - CSF, vacunas, Productos de Imagenología Nuclear).

- Productos que contengan fracciones derivadas de células sanguíneas.

- Parche de Sangre Epidural.

- Gamagrafía de Células Sanguíneas. (Radionucleótidos marcados para localización de sangrado).

- Transplante de células madre (autólogo o alogénico).

- Transplante de órganos.

- Transfusiones alogénicas. (células sanguíneas rojas, células blancas, plaquetas o plasma.

- Donación de sangre autóloga preoperatoria. 
Existen para fines académicos tres momentos claves:

1. Manejo pre-operatorio.

2. Manejo intra-operatorio.

3. Manejo pos-operatorio.

\section{Manejo preoperatorio}

El médico deberá:

a. Elaborar una historia clínica completa enfatizando los antecedentes personales y familiares que sugieran trastornos hemorrágicos e indagando sobre el consumo de fármacos que alteren la hemostasia como la warfarina, aspirina, heparinas, gingo biloba, ajo y productos "naturistas".

b. Identificar las estrategias quirúrgicas y farmacológicas aceptadas por el paciente Testigo de Jehová para ser implementadas en el perioperatorio.

c. Tratar y prevenir patologías que favorezcan estados de anemia.

d. Elevar la hemoglobina preoperatoria a niveles normales.

e. Difundir la información sobre técnicas existentes en el tratamiento de medicina sin sangre.

f. En patología neoplásica considerar la radiología intervencionista para embolización prequirúrgica disminuyendo así el sangrado intraoperatorio.

Dentro de las medidas farmacológicas para elevar la hemoglobina preoperatoria encontramos:

2.1. Eritropoyetina: la hormona Eritropoyetina es un polipéptido glucosilado con 166 aminoácidos y presenta un peso molecular de 34.000 daltons, obtenida por recombinación genética siendo exactamente igual a la humana. Su utilización comienza en el año 1986. Es producida por el riñón en un $90 \%$ y el resto en el hígado. La producción de eritropoyetina es estimulada por la hipoxia celular, censada por las células intersticiales peritubulares de la corteza interna y la médula externa renal. Estimula la diferenciación de los precursores eritroides en la médula ósea. Es importante realizar una valoración interdisciplinaria por parte Medicina Inter-
na-Nefrología, principalmente para aquellos casos en que su uso sea controversial para el balance riesgo-beneficio.

Dosis: se ha propuesto muchos esquemas como estrategia terapéutica en el preoperatorio para la elevación del hematocrito vía subcutánea, tenemos ${ }^{(11)}$ :

1. $300 \mathrm{UI} / \mathrm{kg}$ de peso los días $1,4,7$ y 10 antes de la cirugía.

2. $400 \mathrm{UI} / \mathrm{kg}$ de peso los días $1,5,9$ antes de la cirugía.

3. $600 \mathrm{UI} / \mathrm{kg}$ de peso los días 1 y 10 antes de la cirugía.

4. $600 \mathrm{UI} / \mathrm{kg}$ de peso dos veces por semana, tres semanas antes de la cirugía.

5. $600 \mathrm{UI} / \mathrm{kg}$ de peso los días $7,14,21$ antes de la cirugía.

6. 300-500 UI $/ \mathrm{kg}$ de peso, SC nueve días antes de la cirugía, el día de la cirugía y cuatro días después.

7. $300 \mathrm{UI} / \mathrm{kg}$ de peso diariamente por cuatro días, antes de la cirugía y $150 \mathrm{u} / \mathrm{kg}$ diariamente durante los siguientes siete días, a partir del posoperatorio.

8. $300 \mathrm{UI} / \mathrm{kg}$ de peso por cinco días antes de la cirugía y 150 u/kg los dos días siguientes a la cirugía.

9. $600 \mathrm{UI} / \mathrm{kg}$ de peso una vez por semana, por tres semanas antes de la cirugía.

\section{Contraindicaciones:}

1. Hipertensión arterial no controlada.

2. Hipersensibilidad al principio activo.

3. Primer trimestre del embarazo.

4. Antecedente de evento coronario, cerebrovascular o episodios tromboembólicos en el último año.

\subsection{Hierro $\left.^{(4,} 8,12,13\right)$}

Es uno de los iones necesarios para la formación de hemoglobina. Se estima que de los 4 a 5 gramos de hierro total que hay en el organismo, el $65 \%$ corresponde a la hemoglobina y un $4 \%$ a la mioglobina. Independientemente del esquema implementado de Eritropoyetina ${ }^{(12,13)}$, algunos estudios han demostrado que en los pacientes con depósitos bajos de hierro se genera una eritropoyesis inadecuada caracterizada por aumento de la masa eritrocitaria con formación de eritrocitos hipocrómicos, micro- 
cíticos $^{(3)}$. Pacientes con esquema de eritropoyetina deben contar con un cuadro hemático sistematizado, con índice de reticulocitos y niveles séricos de ferritina con saturación de transferrina; si los niveles de ferritina se encuentran inferiores a $200 \mathrm{ng} /$ $\mathrm{ml}$ o la saturación de transferrina es inferior al $20 \%$ se debe administrar hierro parenteral, de lo contrario se debe iniciar terapia con hierro por vía oral ${ }^{(8)}$.

Los esquemas de administración de Hierro propuestos son ${ }^{(8)}$ :

2.2.1 Dextran hierro: recomendado para administrar los requerimientos totales de hierro en infusión en una solo dosis si es posible. Dosis (ml): 0,0442 (Hb deseada g/ $\mathrm{dL}-\mathrm{Hb}$ actual g/dL) X Peso en $\mathrm{Kg}+(0,26 \mathrm{X}$ Peso $\mathrm{Kg}$ ). La presentación de Dextran Hierro contienen $50 \mathrm{mg}$ de hierro elemental por $\mathrm{ml}$, puede ser administrado por vía intramuscular pero se relaciona con dolor en el sitio de punción y sangrado muscular. La tasa de infusión del Dextran Hierro debe ser inferior a $50 \mathrm{mg} / \mathrm{min}$ realizando previamente una dosis de prueba administrando $0,5 \mathrm{ml}$ ( $25 \mathrm{mg}$ en $50 \mathrm{cc}$ de SSN) y posteriormente se debe monitorizar el paciente durante una hora, con el fin de encontrar posibles reacciones de hipersensibilidad.

\subsubsection{Complejo de Gluconato Férrico: presen-} tación de $125 \mathrm{mg}$ sin diluir, para administrar en una infusión de $12,5 \mathrm{mg} / \mathrm{ml}$ o diluir en 100 cc de SS 0,9\% y administrar en 30 a 60 minutos. Previamente se debe administrar un dosis de prueba de $25 \mathrm{mg}$, principalmente en los pacientes con historia de atopia o reacciones alérgicas medicamentosas.

2.2.3 Carboximaltosa Férrica: se puede administrar en infusión de 15 minutos en dosis que no excedan los $1000 \mathrm{mg}$ de hierro elemental. En personas con historia de atopía o alergia medicamentosa se debe administrar una dosis de prueba de $25 \mathrm{mg}$.

\section{3. Ácido fólico}

Es una vitamina esencial para la diferenciación y maduración de los eritrocitos, específicamente en la síntesis de purinas y de tiamina, necesarias para la formación de ADN. La deficiencia de ácido fólico se caracteriza por la aparición de anemia macrocítica. Se recomienda la ingesta diaria de 100 ucg vía oral y algunos autores recomiendan su uso junto con la eritropoyetina y el hierro ${ }^{(13,14)}$.

\subsection{Vitamina B12}

La maduración de los eritrocitos necesita contar con la presencia de Vitamina B12 para la síntesis de ADN por medio de la formación de fosfato de timidina, uno de los componentes esenciales del ADN. La ausencia de esta vitamina disminuye la formación de ADN con un consecuente fracaso en la división celular, de esta manera se afecta la velocidad de creación de los eritrocitos y se formarán eritrocitos de mayor tamaño denominados macrocitos, de membrana delgada e irregular, propensos a lisis celular ${ }^{(13)}$. Se sugiere la administración diaria a dosis de 500 UCG por día aunque algunos reportes sugieren efectos similares con dosis menores y algunos autores sugieren su uso concomitante con el esquema de eritropoyetina más hierro y ácido fólico(15).

\subsection{Vitamina $C$}

Actúa como agente antioxidante soluble en agua. Recientemente se han encontrado asociaciones sugiriendo que la Vitamina C puede facilitar la movilización de hierro en aquellos tejidos que cuentan con reservas de este ión facilitando la incorporación del hierro en la protoporfirina y génesis de la hemoglobina, especialmente en pacientes que están recibiendo tratamiento con Eritropoyetina. Se sugiere la administración de ácido ascórbico $500 \mathrm{mg}$ vía oral cada 8 horas al menos 2 semanas antes de la intervención quirúrgica ${ }^{(11,13,16)}$.

\section{Manejo intraoperatorio}

El aspecto más importante consiste en que todo el equipo médico-quirúrgico se encuentre adiestrado y sensibilizado hacia 
el objetivo principal: evitar la pérdida sanguínea; esto involucra el ejercicio de un estricto ahorro en la toma de muestras de sangre requeridas para la ejecución de paraclínicos y se incluye la incorporación de técnicas quirúrgicas y farmacológicas avanzadas que nos permitirán ahorrar componentes sanguíneos.

Todo paciente Testigo de Jehová debe llevar un brazalete que informe su negativa a la transfusión de componentes sanguíneos; igualmente su historia clínica debe llevar esta advertencia.

Dentro de las diferentes medidas para ahorro de sangre encontramos:

\subsection{Técnicas quirúrgicas ${ }^{(11,17,18)}$ :}

a. Implementar los principios quirúrgicos Halstedianos basados en el buen trato a los tejidos, estricta hemostasia, disección por planos y ligaduras selectivas.

b. Utilizar dispositivos que disminuyan el sangrado, como el electrocauterio, el láser de Argón, la cirugía endoscópica o video asistida, escalpelo ultrasónico o de radiofrecuencia, pegantes de fibrina y técnicas procoagulantes tópicas.

c. Evitar la pérdida de sangre por la fuerza de gravedad, elevando el área quirúrgica por encima del nivel de la aurícula derecha disminuyendo así la presión hidrostática y sangrado.

d. Uso de torniquetes en cirugías de extremidades y de compresas impregnadas de adrenalina (en una solución de una parte por millón) en los bordes de las heridas.

e. Paciente de cirugía en columna que requiera posición prono, se le debe instaurar rollos a lo largo de ambas líneas medio claviculares, con el fin de disminuir la presión intraabdominal ya que su aumento se transmite sobre la vena cava, aumentando la presión en las venas lumbares y generando mayor sangrado intraoperatorio.

\subsection{Técnicas anestésicas}

El manejo anestésico de los Testigos de Jehová se encuentra encaminado al ahorro de sangre, monitoría estricta hemodinámica instalando una línea arterial y un catéter central ya sea como parte de la monitoría o como acceso a una medida farmacológica. Debemos extremar nuestra atención a cualquier cambio hemodinámico con el fin de mantener la homeostasis, evitar la hipoperfusión tisular e implementar de forma rápida y temprana las medidas correctivas de un eventual choque hipovolémico.

3.2.1 Enfoque del choque hipovolémico: la primera meta es mantener un volumen intravascular adecuado evitando a toda costa la hipoperfusión e hipoxia tisular. Se tolera mucho mejor la anemia que la hipovolemia. Se sugiere:

a. Administración rápida de cristaloides, se recomienda $2000 \mathrm{cc}$

b. Administración rápida de coloides, se recomienda $1000 \mathrm{cc}$

c. Correlación de la administración de cristaloides o coloides con patrón hemodinámico.

d. Apoyo vasopresor farmacológico.

Se debe tener en cuenta que la administración de grandes volúmenes de cristaloides o de coloides pueden ejercer un efecto negativo en las líneas de la coagulación endógena con un efecto deletéreo sobre el sistema hemostático debido a la coagulopatía dilucional y la alteración funcional de las plaquetas ${ }^{(19)}$. Se debe considerar la utilización de Dopamina o Noradrenalina en caso de encontrarse frente a hipotensión que no responda a carga de cristaloides o coloides.

3.2.2 Hipotensión controlada: definida como la disminución de la tensión arterial sistólica hasta llegar a 90 u $80 \mathrm{~mm} / \mathrm{Hg}$ o disminución de la tensión arterial media a valores de 55 a $65 \mathrm{~mm} / \mathrm{Hg}$ en un paciente que se encuentre previamente normotenso. Esta hipotensión se puede inducir con la administración de anestésicos inhalados al aumentar el MAC o con la implementación de fármacos como los nitratos. En las complicaciones de esta técnica podemos encontrar isquemia miocárdica, cerebral u óptica ${ }^{(20,21)}$. Las contraindicaciones de esta técnica son: 
a. Enfermedad cerebrovascular.

b. Enfermedad cardiovascular.

- Enfermedad coronaria.

- Estenosis aórtica.

- HTA no controlada.

- Falla cardiaca.

c. Enfermedad pulmonar severa.

d. Falla renal.

e. Falla hepática.

f. Embarazo.

3.2.3. Técnicas de autotransfusión: implementadas por primera vez en 1818 y establecidas como un ejercicio médico seguro en el año de 1930, las técnicas de autotransfusión son actualmente de utilización activa y resultan de gran utilidad al enfrentar a un paciente Testigo de Jehová con riesgo de sangrado mayor. Se requiere la aceptación del paciente y esta debe ir consignada claramente en el consentimiento informado, en la historia clínica y especialmente en la valoración preanestésica.

Se han descrito clásicamente 3 técnicas:

\subsubsection{Donación de Sangre Autóloga} Preoperatoria (DSP). Esta técnica consiste en la donación de sangre varias semanas antes de la cirugía por parte del mismo paciente para ser reinfundida a futuro en su cirugía. Se requiere del paciente niveles de hemoglobina superiores a $11 \mathrm{~g} / \mathrm{dl}$ o Hematocrito mayor a $33 \%$. Se estima que los pacientes pueden donar hasta $10,5 \mathrm{~mL} / \mathrm{Kg}$, contando las muestras de sangre para la realización de paraclínicos. Las donaciones se pueden programar más de una vez por semana, pero la última donación se debería producir a no menos de 72 horas de la cirugía dando tiempo a la restauración del volumen intravascular y la realización de paraclínicos. Se consideran contraindicaciones a la técnica:

- Evidencia de infecciones o riesgo de bacteremia.

- Cirugía programada para correción de Estenosis Aórtica.

- Angina inestable.

- Ataque epiléptico.

- Infarto agudo de miocardio o accidente cerebrovascular en los últimos 6 meses.

- Enfermedad pulmonar o cardiaca que no ha sido tratada previamente de forma adecuada.

- Enfermedad arterial coronaria de alto grado.

- Enfermedad cardiaca cianozante.

- HTA no controlada.

Existen técnicas combinadas de DSP con administración de eritropoyetina, hierro, vitamina B12 y ácido fólico, para agilizar la recuperación de masa eritrocitaria. Para tal efecto ver el apartado de cada uno de estos medicamentos.

3.2.3.2 Hemodilución Normovolémica Aguda (HNA) . Este método, realizado en

Tabla 3. Donación de sangre autóloga ${ }^{(24)}$

\begin{tabular}{|c|c|}
\hline Ventajas & Desventajas \\
\hline $\begin{array}{l}\text { 1. Previene enfermedades transmitidas } \\
\text { por transfusiones. }\end{array}$ & 1. No disminuye el riesgo de contaminación bacteriana. \\
\hline 2. Previene la aloinmunización por células rojas. & $\begin{array}{l}\text { 2. No disminuye el riesgo de error por incompatibilidad } \\
\text { ABO. }\end{array}$ \\
\hline 3. Complementa el suministro de sangre. & $\begin{array}{l}\text { 3. Es mas costosa que las transfusiones sanguíneas } \\
\text { alogénicas. }\end{array}$ \\
\hline $\begin{array}{l}\text { 4. Proporciona compatibilidad de la sangre } \\
\text { para los pacientes con aloanticuerpos. }\end{array}$ & $\begin{array}{l}\text { 4. Puede resultar en un desperdicio de sangre al no } \\
\text { utilizar los componentes. }\end{array}$ \\
\hline 5. Previene las reacciones adversas por transfusiones. & 5. Aumenta el riesgo de anemia perioperatoria. \\
\hline $\begin{array}{l}\text { 6. Brinda confianza a los pacientes temerosos a las } \\
\text { reacciones adversas de las transfusiones. }\end{array}$ & 6. Lesión de la sangre por almacenamiento. \\
\hline
\end{tabular}


salas de cirugía previo al inicio del procedimiento quirúrgico, consiste en extraer una determinada cantidad de sangre del paciente y reemplazarla por fluidos intravenoso con el fin de mantener un volumen circulatorio normal con aporte de oxígeno adecuado a los tejidos. Esta técnica crea una anemia que puede poner al paciente en estado de hipoxia tisular. En el transcurso de la cirugía se realiza una juiciosa cuantificación de las perdidas sanguíneas permisivas y una vez se alcancen estas o se supere el momento crítico de la cirugía donde se encuentra la mayor probabilidad de sangrado, se reinfunde la sangre que previamente había sido extraída. Por ningún motivo la sangre extraída debe salir de la sala de cirugía; esta además es una frecuente exigencia de los pacientes Testigos de Jehová para poder implementar la técnica ${ }^{(22,23)}$.

Durante el proceso de hemodilución, el estado de normovolemia se mantiene administrando cristaloides y coloides; Se reemplaza $1 \mathrm{ml}$ de sangre por $1 \mathrm{ml}$ de coloides o por $3 \mathrm{ml}$ de cristaloides. La fórmula para calcular que cantidad de sangre puede ser retirada utilizando la técnica de hemodilución normovolémica aguda es ${ }^{(24)}$ :

$$
\mathrm{V}=\mathrm{VS} \times(\mathrm{Hi}-\mathrm{Hf}) / \mathrm{PHif}
$$

V: Volumen a extraer.

VS: Volumen sanguíneo estimado (Volemia).

Hi: Hematocrito inicial (el reporte de hematocrito del último cuadro hemático).

Hf: Hematocrito final (hematocrito mínimo que se puede permitir con la extracción de la sangre).

PHif:Promedio de hematocritos (entre hematocrito inicial y hematocrito final).

Condiciones para realizar la $\mathrm{HNA}^{(24)}$ :

- Cirugía con altas probabilidades de transfusión perioperatoria.

- Hemoglobina preoperatoria por encima de $12 \mathrm{~g} / \mathrm{dl}$.

- Ausencia de infección.

- Pacientes sin riesgo de bacteremia.

Ventajas de la HNA:

- Mejora la reología sanguínea al producir hemodilución.

- Conserva la función plaquetaria si es reinfundida antes de 6 horas.
- Disminuye la pérdida de masa eritrocítica intraoperatoria al disminuir el hematocrito.

- Más económica y fácil de implementar respecto a la donación preoperatoria.

- Comparte las ventajas de la donación preoperatoria de sangre.

- La sangre no sufre lesión por almacenamiento.

- Disminuye la hipotermia respecto a transfundir sangre de banco.

3.2.3.3 Recolección y reinfusión de Eritrocitos - Salvador de Células . Esta técnica se caracteriza por recolectar la sangre del campo operatorio, seleccionar los eritrocitos, lavarlos y empacarlos para administrarlos al paciente. La recolección y reinfusión de eritrocitos es una técnica implementable en el intraoperatorio y el posoperatorio; comparte las mismas ventajas y desventajas que la Hemodilución Normovolémica Aguda(22). Para la implementación de esta técnica se requiere la disposición de equipos que no son descritos en esta revisión y que son de frecuente utilización en cirugía cardiovascular y trasplante hepático. Entre sus complicaciones podemos tener embolismo aéreo y error en las mezclas de las soluciones implementadas en los equipos Salvador de Células que usualmente requieren SSN, glicina o agua estéril.

\subsection{Técnicas farmacológicas}

Hay fármacos que actúan sobre los procesos hemostáticos y que usualmente los pacientes Testigos de Jehová aceptan. A continuación se realiza una descripción de los principales de ellos.

3.3.1. Complejo protombinico: el complejo concentrado de protrombina es utilizado ampliamente con el fin de revertir rápidamente los efectos de los anticoagulantes que actúan inhibiendo la biosíntesis de factores vitamina $\mathrm{K}$ dependientes (II, VII, IX, X) de los pacientes que toman warfarina para la prevención de eventos tromboembólicos o fármacos como el acenocumarol o el fenocumarol. Su administración reduce efectivamente el valor del INR. También se utiliza en pacientes que cursan con sangrado 
mayor. Entre las contraindicaciones se encuentran la hipersensibilidad al medicamento, riesgo de coagulación intravascular diseminada, enfermedad tromboembólica arterial y trombocitopenia por heparina $(27,28)$.

Se dosifica de acuerdo al valor del INR. $\mathrm{Si}$ este es mayor a 5 se recomienda la administración de $30 \mathrm{UI} / \mathrm{kg}$, si el INR es menor a 5 la dosis a administrar es de $15 \mathrm{UI} / \mathrm{kg}$. Se infunde a una velocidad de $1 \mathrm{~mL} /$ minuto y no se recomienda la mezcla con otros medicamentos. Su latencia es de 10 minutos y se recomienda la administración concomitante de Vitamina $K^{(27)}$.

3.3.2 Vitamina $K$ : hay factores de la coagulación dependientes de vitamina $\mathrm{K}$. Estos factores son el II, VII, IX y X. La vitamina K se sintetiza de manera continua por las bacterias del tubo digestivo. Se recomienda la administración de vitamina $\mathrm{K}$ de forma concomitante con el Complejo Protrombínico. Dosis $(13,27)$ :

- Vía oral 10 mg día dividido cada 12 horas.

- Vía intravenosa se puede administrar 10 mg en infusión durante 20 a 60 minutos y se puede repetir la dosis a las 12 horas. No se recomienda la administración subcutánea o intramuscular por el alto riesgo a formar hematoma.

\section{Contraindicaciones:}

- Contraindicada en los pacientes alérgicos a las vitaminas $\mathrm{k}$.

- Deficiencia de la glucosa-6-fosfatodeshidrogenasa (riesgo de anemia hemolítica).

- Se administrará con precaución en pacientes con insuficiencia renal o cuando se sospeche patología trombótica.

- Administrar con precaución en pacientes con enfermedad hepática, alcoholismo, epilepsia, mujeres embarazadas y niños.

3.3.3 Análogos de la lisina: son los medicamentos más utilizados en clínica. Normalmente la lisina se une a un sitio específico del plasminógeno para su transformación en plasmina y así degradar la fibrina. Esto fármacos actúan en la unión del plasminógeno a la fibrina mediante el sitio dependiente de lisina inhibiendo su acción y por tanto la fibrinólisis. Los medicamentos que pertenecen a este grupo son el ácido tranexámico y el ácido amino caproico.

3.3.3.1 Ácido tranexámico. Este medicamento implementado ampliamente en diferentes escenarios que involucran hemorragias, es un fármaco sintético, derivado del amino ácido de lisina que ejerce su efecto antifibrinolítico al bloquear de manera reversible la lisina en los sitios de unión en las moléculas de plasminógeno. Clásicamente se administra en bolo de carga de $10 \mathrm{mg} / \mathrm{kg}$ y continuando la infusión a $1 \mathrm{mg} / \mathrm{kg}$ hora ${ }^{(25)}$.

3.3.3.2 Ácido epsilon amino caproico. Al igual que el ácido tranexámico, el ácido epsilon amino caproico comparte el mismo mecanismo de acción, de esta manera se evita la transformación del plasminógeno a la plasmina y se evita su acción proteolítica sobre la fibrina consiguiendo así la estabilización del coagulo. Las dosis en adultos oscila entre 10 a $30 \mathrm{gr}$ y en la población pediátrica de 80 a $160 \mathrm{mg} / \mathrm{Kg}$ continuando la infusión a $15 \mathrm{mg} / \mathrm{kg} / \mathrm{h}^{(25,26)}$.

3.3.3.3 Aprotinina. Es un inhibidor no selectivo de proteasas, principalmente las que se encuentran involucradas en la cascada de la coagulación que actúa sobre los residuos de serina de las proteasas; no se considera la utilización de aprotinina por estar en el momento suspendida del mercado desde 2007 al encontrarse asociada al aumento de mortalidad en cirugía cardiovascular según un estudio canadiense.

3.3.4 Desmopresina ${ }^{(29)}$ : es un análogo de la vasopresina que ejerce un efecto hemostático mediante la inducción y expresión del factor Von Willebrand (FVW) desde sus lugares de almacenamiento en las células endoteliales. Ha demostrado ser útil en el tratamiento o prevención de episodios hemorrágicos en pacientes con enfermedad de Von Willebrand, hemofilia A y defectos de la función plaquetaria. Suministra un nivel satisfactorio en la hemostasia reduciendo el uso de hemoderivados a pesar de no 
conocer concretamente el mecanismo de acción. Se cree que en el sangrado activo juega un papel relacionado con la formación y expresión del FVW acelerando la activación del Factor X; inusualmente la desmopresina induce la expresión de moléculas de FVW más grandes que poseen mayor afinidad por las plaquetas. Las dosis sugeridas intranasal (Puff) es de $150 \mathrm{ucg} / \mathrm{Kg}$ llegando hasta los $50 \mathrm{~kg}$. Para mayores de $50 \mathrm{~kg}$ administrar $300 \mathrm{ucg} / \mathrm{Kg}$. Intravenoso: $0,3 \mathrm{ucg} / \mathrm{kg}$ diluido en SSN (15-30 ml para niños, 50-100 ml para adultos) y administrar en 15 a $30 \mathrm{mi}-$ nutos. Subcutáneo: $0,3 \mathrm{ucg} / \mathrm{kg}$ de peso, dosis única.

Con la administración de Desmopresina como efectos adversos se pueden encontrar taquicardia, flushing, cefalea e hiponatremia.

\section{Contraindicaciones}

- Enfermedad ateroesclerótica.

- Trombosis venosa.

- Púrpura trombocitopénica.

- No administrar en pacientes con enfermedad de Von Willebrand tipo 2B.

3.3.5 Factor VII Activado (FVIIa): clásicamente la coagulación se dividió en la vía extrínseca y la vía intrínseca. Hoy en día es mejor entenderla como el equilibrio del proceso de formación del coágulo (trombogénesis) y la destrucción del coágulo (fibrinolísis). El primero de estos procesos es mejor entendido como los procesos de activación, amplificación y propagación donde la actividad plaquetaria es el eje. El FVIIa hace parte de la clásica vía extrínseca siendo el encargado de iniciar la cascada procoagulante al activar los factores IX y X. La administración de FVIIa ayuda a la génesis de una adecuada hemostasia proporcionando soporte en aquellos individuos en donde la vía intrínseca se encuentra lesionada. Se recomienda la administración a dosis variables que van desde de 90 hasta $200 \mathrm{ucg} / \mathrm{Kg}^{(30)}$.

3.3.6. Transportadores sintéticos de oxígeno y perfluocarbonos ${ }^{(31)}$ : si bien este tipo de terapia farmacológica aún se encuentra en fase de estudio para poder ser implementadas de forma generalizada, se encuentran reportes de casos que hablan a favor de estos medicamentos. Se mantiene como una alternativa futura a la transfusión de eritrocitos.

\section{Cuidados posoperatorios}

a. Si se ha configurado sangrado mayor con o sin choque hipovolémico es prudente manejar estos pacientes en UCI para vigilancia hemodinámica y de la coagulación estricta.

b. Debe garantizarse en el posoperatorio la vigilancia permanente hemodinámica y de drenajes y sitios de sangrado para detectar tempranamente un resangrado postoperatorio e iniciar las medidas correctivas.

c. La hipotensión permisiva, controlada y con estricta monitoría sería prudente si hay sangrado persistente leve. Se debe asegurar que o existe contraindicación para esta terapia.

d. La reinfusión posoperatoria de sangre recolectada por tubos torácicos, mediastinales o sistemas de drenaje en cirugía como la ortopédica, puede realizarse de manera segura en este momento.

e. Si hay resangrado debe considerarse el uso de técnicas de cirugía de control de daños como empaquetamientos y embolizaciones selectivas).

f. Profilaxis con inhibidores de la bomba de protones o protectores de mucosa gástrica, para evitar las úlceras por estrés.

g. Sedación y analgesia suficiente para disminuir el consumo y demanda de oxígeno.

h. Profilaxis antiemética suficiente para evitar el pujo y aumento de presiones de cavidades que puedan llevar a resangrados.

En todo caso, el gran armamentario de opciones que se tienen desde el pre hasta el posoperatorio para realizar "cirugía sin sangre", debe ser individualizado a cada paciente y circunstancia particular.

\section{Bibliografía}

1. The Royal College of Surgeons of England. 2002. Code of practice for the surgical management of Jehovah's witnesses 
Published by the Royal College of Surgeons of England.

2. ¿Cómo puede salvarle la vida la sangre? 1990. Watch Tower Bible and Tract Society Pennsylvania, New York Inc. Revista Atalaya.

3. Jiménez C.E. 2005. Recomendaciones medicoquirúrgicas para disminuir el uso y pérdida de derivados sanguíneos. Revista Colombiana de Cirugía. 20(2).

4. Nash M.J. 2004. Hannah Cohen Management of Jehovah's Witness patients with haematological problems. Blood Reviews 18:211-217.

5. Beauchamp T, Childress J. F. 1999. Principios de ética biomédica. Masson, S.A. Barcelona. http://intensivos.uninet.edu/03/ 0301.html.

6. Sentencia T-659/02 Referencia: expediente T589908. Bogotá, D. C., quince (15) de agosto de dos mil dos (2002).

7. British Committee for Standards in Haematology: Writing Group: D. Stains by, S. MacLennan, D. Thomas, J. Isaac and P. J. Hamilton 4 Guide lines on the management of massive blood loss. doi:10.1111/j.13652141.2006.06355.x Journal Compilation a 2006 Blackwell Publishing Ltd, British Journal of Haematology, 135:634-641.

8. Kenrick Berend, MD, Ph D, Marcel Levi, MD, PhD. 2009. Management of Adult Jehovah's Witness Patients with Acute Bleeding. The American Journal of Medicine 122:1071-1076.

9. Donat R. Spahn, Vladimir Cerny, Timothy J Coats, Jacques Duranteau, Enrique Fernández- Mondéjar, Giovanni Gordini, Philip F Stahel, Beverley J Hunt, Radko Komadina, Edmund Neugebauer, Yves Ozier, Louis Riddez, Arthur Schultz, JeanLouis Vincent and Rolf Rossaint. 2007. Management of bleeding following major trauma: a European Guideline. Published: 13 Feb 2007. Critical Care 11:R17 (doi:10.1186/ cc5686) This articles on line at: http:// ccforum.com/content/11/1/R17

10.Zenon M. Bodnaruk, Colin J. Wong, and Mervyn J. Thomas. 2004. Meeting the Clinical Challenge of Care for Jehovah's Witnesses Transfusion Medicine Reviews, 18(2):105-116.

11. Jiménez J. CE. 2005. Recomendaciones médico-quirúrgicas para disminuir el uso y pérdida de derivados sanguíneos. Revista Colombiana de Cirugía. 20(2).

12. Mcdougall IC, Ashenden M. 2009. Current and up comin gery thro poiesis-stimulatin gagents, iron products, and other novel anemia medications. Adv. Chronic Kidney Dis 16(2):117-130.

13. Guyton.
14. Omar Dary. 2009. Nutrition Science Policy Nutrition all interpretation of folic acid interventions. International Life Sciences Institute. Nutrition Reviews@ 67(4):235-244.

15. Patrick J. Stover. 2010. Vitamin B12 and older adults. Current Opinion in Clinical Nutrition and Metabolic Care 13:24-27.

16. Tarng DC. 2007. Novel aspects of vitamin C in epoetin response. J Chin Med Assoc 70(9):357-360.

17. Faris P, Spende K, Larholt M. 1999. The predictive power of base line hemoglobin for transfusion risk in surgery patients. Orthopedics 22(suppl):s135-s140.

18. Macintyrea. 1992. Blood transfusion and hemostatic management in the perioperative period. Can J Anaesth 39: Pt 2, R101-114.

19. De Jonge E, Levi M. 2001. Effects of different plasma substitutes on blood coagulation: a comparative review. Crit Care Med. 29:12611267.

20. Purdham RS. 1996. Reduced blood loss with hemodynamic stability during controlled hypotensive anesthesia for Lefort I. Maxillary osteotomy using high dose fentanyl: a retrospective study. CRNA 33-46.

21. Jones R, Moulton M. 1998. Central venous pressure and its effect on blood loss during liver resection. Br J Surg 85:1058-1060.

22. Jonathan H. Waters, MD. 2005. Red Blood Cell Recovery and Reinfusion Anesthesiology Clinics of North America 23:283-294.

23. Terri G. Monk, MD. 2005. Acute Normovolemic Hemodilution Anesthesiology Clin $N$ Am 23:271-281.

24. Lawrence T. Goodnough, MD. 2005. Autologous Blood Donation Anesthesiology Clin N Am 23:263-270.

25. Christopher J. Dunn andKaren L. Goa. 1999. Tranexamic Acid A Review of its Use in Surgery and Other Indications. Adis International Limited, Auckland, New Zealand Drugs 57(6):1005-1032.

26. David Henry MB Ch.B, Paul Carless BH Sc M Med Sc (ClinEpid), Dean Fergusson PhD MHA, Andreas Laupacis MD MSc. 2009. The safety of aprotinin and lysine-derived antifibrinolytic drugs in cardiac surgery: a metaanalysis. CMAJ 180(2).

27. Kerstin S Schick, Jan M Fertmann, KarlWalter Jauchand Johannes N Hoffmann. 2009. Prothrombin complex concentrate in surgical patients: retrospective evaluation of vitamin $\mathrm{K}$ antagonis treversa land treatment of severe bleeding. Critical Care 13:R191(doi:10.1186/cc8186).

28. Cindy A. Leissinger, Philip M. Blatt W. 2008. Role of prothrombin complex concentrates in reversing warfarin anticoagulation: A 
review of the literature. Keith Hoots and Bruce Ewenstein Am J Hematol 83:137-143.

29. Bulent O, Zgonenel, Madhvi Rajpurkar, Jeanne M Lusher. 2007. How do you treat bleeding disorders with desmopressin?. Postgrad Med J 83:159-163. doi: 10.1136/ pgmj.2006.052118.

30. Nigel Mackman, PhD. 2009. The Role of Tissue Factor and Factor VIIa in Hemostasis. From the Division of Hematology/Oncology, Department of Medicine, University of North
Carolina at Chapel Hill, Chapel Hill, North Carolina. Anesth Analg. 108(5):1447-1452. doi:10.1213/ane. 0b013e31819bceb1.

31. Paula F. Moon-Massat, DVM, Aryeh Shander, MD, Mazyar Javidroozi, MD, and A. Gerson Greenburg. 2010. When Bloodis Notan Option: Factors Affecting Survival After the Use of a Hemoglobin-Based Oxygen Carrier in 54 Patients with Life-Threatening Anemia Colin F. Mackenzie, MB ChB, FRCA, FCCM, ${ }^{*} \mathrm{MD}$ 110(3). www.anesthesia-analgesia.org 
